\title{
Friction losses of Newtonian and non-Newtonian fluids flowing in laminar regime in a helical coil
}

\author{
T.A. Pimenta, J.B.L.M. Campos
}

\begin{abstract}
A B S T R A C T
This study aimed to carry out experimental work to determine, for Newtonian and non-Newtonian fluids, the friction factor $\left(f_{c}\right)$ with simultaneous heat transfer, at constant wall temperature as boundary condition, in fully developed laminar flow inside a vertical helical coil. The Newtonian fluids studied were aqueous solutions of glycerol, $25 \%, 36 \%, 43 \%, 59 \%$ and $78 \%(w / w)$. The non-Newtonian fluids were aqueous solutions of carboxymethylcellulose (CMC), a polymer, with concentrations of $0.2 \%, 0.3 \%, 0.4 \%$ and $0.6 \%(\mathrm{w} / \mathrm{w})$ and aqueous solutions of xanthan gum (XG), another polymer, with concentrations of $0.1 \%$ and $0.2 \%(w / w)$. According to the rheological study done, the polymer solutions had shear-thinning behavior and different values of viscoelasticity. The helical coil used has an internal diameter, curvature ratio, length and pitch, respectively: $0.00483 \mathrm{~m}, 0.0263,5.0 \mathrm{~m}$ and $11.34 \mathrm{~mm}$. It was concluded that the friction factors, with simultaneous heat transfer, for Newtonian fluids can be calculated using expressions from literature for isothermal flows. The friction factors for CMC and XG solutions are similar to those for Newtonian fluids when the Dean number, based in a generalized Reynolds number, is less than 80 . For Dean numbers higher than 80 , the friction factors of the CMC solutions are lower those of the XG solutions and of the Newtonian fluids. In this range the friction factors decrease with the increase of the viscometric component of the solution and increase for increasing elastic component. The change of behavior at Dean number 80, for Newtonian and non-Newtonian fluids, is in accordance with the study of Ali [4]. There is a change of behavior at Dean number 80, for Newtonian and non-Newtonian fluids, which is in according to previous studies. The data also showed that the use of the bulk temperature or of the film temperature to calculate the physical properties of the fluid has a residual effect in the friction factor values.
\end{abstract}

Keywords:

Helical coil

Friction factor

Heat transfer

Newtonian fluids

Non-Newtonian fluids

Laminar flow

\section{Introduction}

Coiled tubes of helical shape are widely used as heat exchangers and have application in various industries: chemical, biological, petrochemical, mechanical, biomedical among others. In these industries, they are used in a large range of processes: sterilization, pasteurization, concentration, crystallization, separation (distillation) and reaction. They are also used in general purpose equipment, such as refrigeration, air conditioning and water heating. The fluids involved in these processes may have Newtonian or non-Newtonian behavior.

The helical coils are widely used because they have large heat transfer areas, they are compact, and above all, the geometry promotes good mixing of the fluids increasing the heat and mass

\footnotetext{
* Corresponding author. Address: Rua António Bernardino de Almeida n 431, 4200-072 Porto, Portugal. Tel.: +351 228340500; fax: +351 228321159.
}

transfer coefficients. In addition, this equipment is low cost and of easy construction and maintenance.

The mixing characteristics are consequence of the development of secondary flows along the tube, the so called Dean effect [1]. These secondary flows appear due to the centrifugal force acting on the fluid elements. The difference in axial velocity among the fluid elements flowing in a cross-section leads to a centrifugal gradient. Those elements flowing in the center are projected, under the centrifugal force, into the outer wall direction of the coil, where they suffer a decrease of velocity. Afterwards, they return to the center of the tube, forming two, for the case of tubes with a circular cross-section, counter-rotation vortices, i.e., Dean cells [1]. This flow pattern promotes the mixture of the fluid elements. Studies have shown that these secondary flows still have a stabilizing effect on the global flow, promoting a higher critical Reynolds number for transition, from laminar to turbulent, than that in a straight tube.

There are several studies in the literature concerning the friction losses in helical coils for Newtonian and non-Newtonian 


\begin{tabular}{|c|c|c|c|}
\hline \multicolumn{4}{|c|}{ Nomenclature } \\
\hline$a_{T}$ & shift factor & $T_{t i}$ & internal surface temperature of the top wall of the agi- \\
\hline$C_{p}$ & heat capacity of the fluid $\left(\mathrm{J} \mathrm{kg}^{-1} \mathrm{~K}^{-1}\right)$ & & tation tank $\left({ }^{\circ} \mathrm{C}\right)$ \\
\hline$C_{\text {pwater }}$ & heat capacity of the water $\left(\mathrm{J} \mathrm{kg}^{-1} \mathrm{~K}^{-1}\right)$ & $T_{w}$ & internal wall temperature of the coil $\left({ }^{\circ} \mathrm{C}\right)$ \\
\hline$C_{\text {psyst }}$ & mean heat capacity of the system (oil, coil and tank) & & mean velocity of the fluid $\left(\mathrm{m} \mathrm{s}^{-1}\right)$ \\
\hline & $\left(\mathrm{J} \mathrm{kg}-1 \mathrm{~K}^{-1}\right)$ & $(U A)_{\text {losses }}$ & global heat transfer coefficient from the liquid to \\
\hline $\begin{array}{l}d_{c} \\
d_{c} / d_{i}\end{array}$ & $\begin{array}{l}\text { coil diameter }(\mathrm{m}) \\
\text { curvature of the coil }\end{array}$ & & $\begin{array}{l}\text { the ambient air times the internal area of the tank } \\
\left(\mathrm{W} \mathrm{K}^{-1}\right)\end{array}$ \\
\hline$d_{e q}$ & equivalent diameter of the coil (m) & $\Delta P$ & pressure drop of the fluid in the coil $(\mathrm{Pa})$ \\
\hline$d_{i}$ & inside tube diameter $(\mathrm{m})$ & & \\
\hline$f$ & fanning friction factor of Newtonian and non-Newto- & \multicolumn{2}{|c|}{ Greek symbols } \\
\hline & $\begin{array}{l}\text { nian fluids flowing inside a straight tube for laminar re- } \\
\text { gime }\end{array}$ & $\begin{array}{l}\gamma \\
\dot{\gamma}\end{array}$ & $\begin{array}{l}\text { strain } \\
\text { shear rate }\left(\mathrm{s}^{-1}\right)\end{array}$ \\
\hline$f_{c}$ & fanning friction factor of Newtonian and non-Newto- & $\eta$ & viscometric viscosity (Pa s) \\
\hline & nian fluids flowing inside of the coil for laminar regime & $\lambda$ & relaxation time $(\mathrm{s})$ \\
\hline$G^{\prime}$ & storage module $(\mathrm{Pa})$ & $\rho$ & density $\left(\mathrm{kg} \mathrm{m}^{-3}\right)$ \\
\hline$G^{\prime \prime}$ & loss module $(\mathrm{Pa})$ & $\omega$ & angular frequency $\left(\mathrm{s}^{-1}\right)$ \\
\hline$K$ & consistency index of the fluid $\left(\mathrm{Pa} \mathrm{s}^{\mathrm{n}}\right)$ & & \\
\hline$k_{f}$ & thermal conductivity of the fluid ( $\mathrm{W} \mathrm{m} \mathrm{K} \mathrm{K}^{-1}$ ) & \multicolumn{2}{|c|}{ Dimensionless numbers } \\
\hline$L_{\text {coil }}$ & length of the coil (m) & De & Dean number \\
\hline$m_{\text {syst }}$ & mass of oil, coil and tank (kg) & $\mathrm{De}_{(g)}$ & modified Dean number \\
\hline$\dot{m}^{3 y s}$ & mass flow inside the coil $\left(\mathrm{kg} \mathrm{s}^{-1}\right)$ & $\mathrm{Eu}$ & Euler number \\
\hline$m_{\text {water }}$ & mass flow of water inside the coil $\left(\mathrm{kg} \mathrm{s}^{-1}\right)$ & $\mathrm{G}_{\mathrm{rhc}}$ & geometric number \\
\hline$n$ & power-law index of the fluid (index of behavior) & $\mathrm{He}$ & Helical number \\
\hline$n_{t}$ & number of turns of the coil & $\operatorname{Pr}$ & Prandtl number \\
\hline$p$ & pitch of the coil (m) & $\operatorname{Pr}_{(g)}$ & modified Prandtl number \\
\hline$P$ & pressure $\left(\mathrm{N} \mathrm{m}^{-2}\right)$ & $\operatorname{Pr}_{w}$ & Prandtl number at wall temperature of the tube \\
\hline$t$ & time $(\mathrm{s})$ & $\operatorname{Re}$ & Reynolds number \\
\hline$T$ & temperature $(\mathrm{K})$ & $\operatorname{Re}_{c}$ & critical Reynolds number \\
\hline$T_{0}$ & reference temperature $(\mathrm{K})$ & $\operatorname{Re}_{c}^{I}, \operatorname{Re}_{c}^{I I}$ & critical Reynolds numbers $I, I I$ \\
\hline$T_{a m b}$ & ambient temperature $\left({ }^{\circ} \mathrm{C}\right)$ & $\operatorname{Re}_{g}$ & generalized Reynolds number \\
\hline$T_{e}$ & inlet temperature of the fluid in the coil $\left({ }^{\circ} \mathrm{C}\right)$ & Wi & Weissenberg number \\
\hline$T_{f}$ & mean film temperature of the fluid $\left({ }^{\circ} \mathrm{C}\right)$ & & \\
\hline$T_{l e}$ & external surface temperature of the lateral wall of the & \multicolumn{2}{|l|}{ Acronyms } \\
\hline & agitation $\operatorname{tank}\left({ }^{\circ} \mathrm{C}\right)$ & CMC & carboxymethylcellulose \\
\hline$T_{l i}$ & internal surface temperature of the lateral wall of the & PAA & polyacrylamide \\
\hline & agitation tank $\left({ }^{\circ} \mathrm{C}\right)$ & PEO & polyethylene oxide \\
\hline$T_{m}$ & mean temperature of the fluid (bulk temperature) $\left({ }^{\circ} \mathrm{C}\right)$ & $\mathrm{MHC}$ & modified hart correlation \\
\hline$T_{\text {oil }}$ & bulk oil temperature $\left({ }^{\circ} \mathrm{C}\right)$ & $\mathrm{w} / \mathrm{w}$ & percentage by weight \\
\hline$T_{s}$ & exit temperature of the coil $\left({ }^{\circ} \mathrm{C}\right)$ & XG & xanthan gum \\
\hline$T_{t e}$ & $\begin{array}{l}\text { external surface temperature of the top wall of the agi- } \\
\text { tation tank }\left({ }^{\circ} \mathrm{C}\right)\end{array}$ & & \\
\hline
\end{tabular}

fluids. The friction factor for a fluid, Newtonian or non-Newtonian, flowing in a helical coil is larger than that for the same fluid flowing in a straight tube under the same conditions. Zhou and Shah [2] and Naphon and Wongwises [3] did extensive and critique reviews of theoretical and experimental studies about flow in curved pipes, Newtonian and non-Newtonian fluids, single and also diphasic flows. Reviewing these works, it is straightforward that there is a limited knowledge concerning the friction factor for nonNewtonian fluids flowing in coils, in particular about the effect of the viscoelasticity of the fluid. In the case of Newtonian fluids, there are still aspects not yet fully understood, including geometric effects and classification of flow regimes [4,5].

Most of the frictional pressure drop studies of fluids flowing inside helical coils have been performed in isothermal conditions. However, coils are used in heat exchange equipment. The main difference in the frictional pressure drop between isothermal and non-isothermal flows comes from the change of the velocity profile by temperature gradient effects. Seban and McLaughlin [6] conducted experiments to determine the friction factor for Newtonian fluids, in laminar and isothermal flows and also with simultaneously heat transfer for the condition of constant heat flux in the wall. To calculate the physical properties, these authors used the mean temperature of the film, $T_{f}$, and they obtained similar results for isothermal and non-isothermal flow conditions. Rogers and Mayhew [7] performed identical studies but under the condition of constant wall temperature. These authors presented the following expression which relates the frictions factors for isothermal, $f_{c}^{I}$, and non-isothermal, $f_{c}^{N I}$, flows:

$f_{c}^{I}=f_{c}^{N I}\left(\operatorname{Pr} / \operatorname{Pr}_{w}\right)^{1 / 3}$

where $\operatorname{Pr}$ and $\operatorname{Pr}_{w}$, are, respectively, the Prandtl numbers calculated at the bulk temperature of the fluid and at the inner wall temperature of the coil.

Schmidt [8] also studied the pressure drop for laminar flow of Newtonians fluids inside helical coils for isothermal and non-isothermal conditions under constant wall temperature. He concluded that the physical properties should be determined at the mean film temperature.

The conclusions of the different studies, in particular for Newtonian fluids, are multiple and, sometimes divergent, as is the case of the works of Ali [4] and Cioncolini and Santini [5] which refer the existence of two sub-regimes in the laminar flow. 
Besides the intention to clarify these divergent conclusions for Newtonian fluids, the main objective of the present study is to find accurate pressure drop correlations for non-Newtonian fluids flowing in helical coils with simultaneous heat transfer and particularly to study the contribution of the elastic component.

The next item describes a detailed literature review, with particular emphasis on the work done under identical conditions of the present study.

\subsection{Review for Newtonians fluids}

The expressions that are listed, chronologically, in Table 1 allow the calculation of the friction factors for laminar flow, isothermal and fully developed Newtonian fluids circulating inside helical coils, $f_{c}$. Most of these expressions are expressed in terms of the friction factor for laminar flow in straight tubes $(f=16 / \mathrm{Re})$.

In the table De represents the Dean number given by:

$\operatorname{De}=\operatorname{Re}\left(d_{i} / d_{c}\right)^{1 / 2}$

where Re is the Reynolds number, $\operatorname{Re}=\rho v d_{i} / \eta$, and $v, \rho$, and $\eta$ are, respectively, the mean velocity, the density and the viscometric viscosity of the fluid.

\subsection{Review for non-Newtonians fluids}

For non-Newtonian fluids in helical coils, some of the studies, among the few in the literature, about friction factor in fully developed laminar flows are presented in the Table 2.

The friction factor in a straight tube for fully developed laminar flow of non-Newtonian fluids, which follow the power law, is given by the same expression of Newtonian fluids, but based in the generalized Reynolds number of Metzner [19], $f=16 / \operatorname{Re}_{g}$ :

$\operatorname{Re}_{g}=\frac{d_{i}^{n} v^{2-n} \rho}{K\left(\frac{1+3 n}{4 n}\right)^{n} 8^{n-1}}$ where $K$ and $n$ are, respectively, the consistency index and the behavior index of the fluid.

\section{Experimental}

\subsection{Experimental set-up}

Table 3 presents the dimensions of the copper coil used in this study. Fig. 1 shows a flow diagram of the experimental set-up used to determine the friction losses, with simultaneous heat transfer, for the condition of constant wall temperature. The fluid circulated in a close loop from a first tank, where the temperature was controlled and set constant at $20^{\circ} \mathrm{C}$ by means of a heat pump and a refrigerator, to inside the copper coil. The coil was submerged in a bath of oil mechanically agitated placed in another tank as depicted in Fig. 1. This tank was thermally isolated and was provided with a heating system and a temperature controller. The experimental set-up had a centrifugal pump, a transducer to measure the pressure drop of the fluid inside the helical coil (uncertainty $0.028 \%$ full scale (0-6 bar)), an electromagnetic flowmeter (uncertainty of $1.2 \%$ of the volumetric flow), several thermometers $\mathrm{T}$ type and Pt100 type (maximum uncertainty of $0.3^{\circ} \mathrm{C}$ ) and a data acquisition system (OMEGA PCI 1602 and Validyne UPC601-T).

The oil, where the coil was immersed, was a mineral oil used for heat transfer, and it was accompanied by a technical sheet with the physical properties.

The heat transfer boundary condition was constant wall temperature $\left(T_{w}\right)$ due to the high rotational velocity of the stirrer (1100 $\mathrm{min}^{-1}$ ) and, by consequence, to the low heat transfer resistance from the bath to the internal wall of the coil. This boundary condition was validated comparing temperatures measured at the wall of the coil (two positions) and at the oil bath. The relative difference was never higher than $4 \%$.

The experimental rig was validated calculating, with experimental data, both sides of the energy balance equation applied to

Table 1

Literature pressure drop correlations for Newtonian fluids.

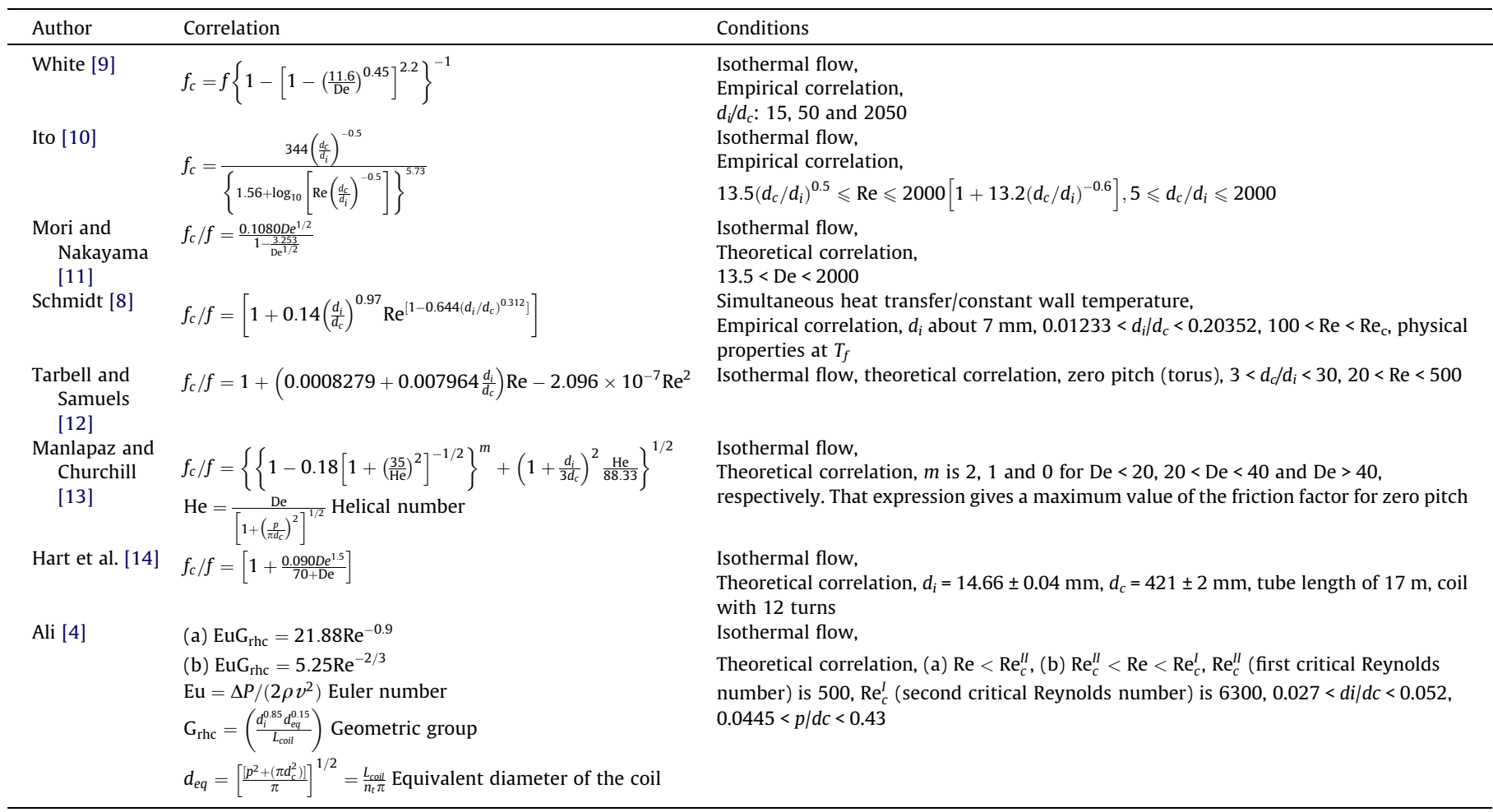


Table 2

Literature pressure drop correlations for non-Newtonian fluids.

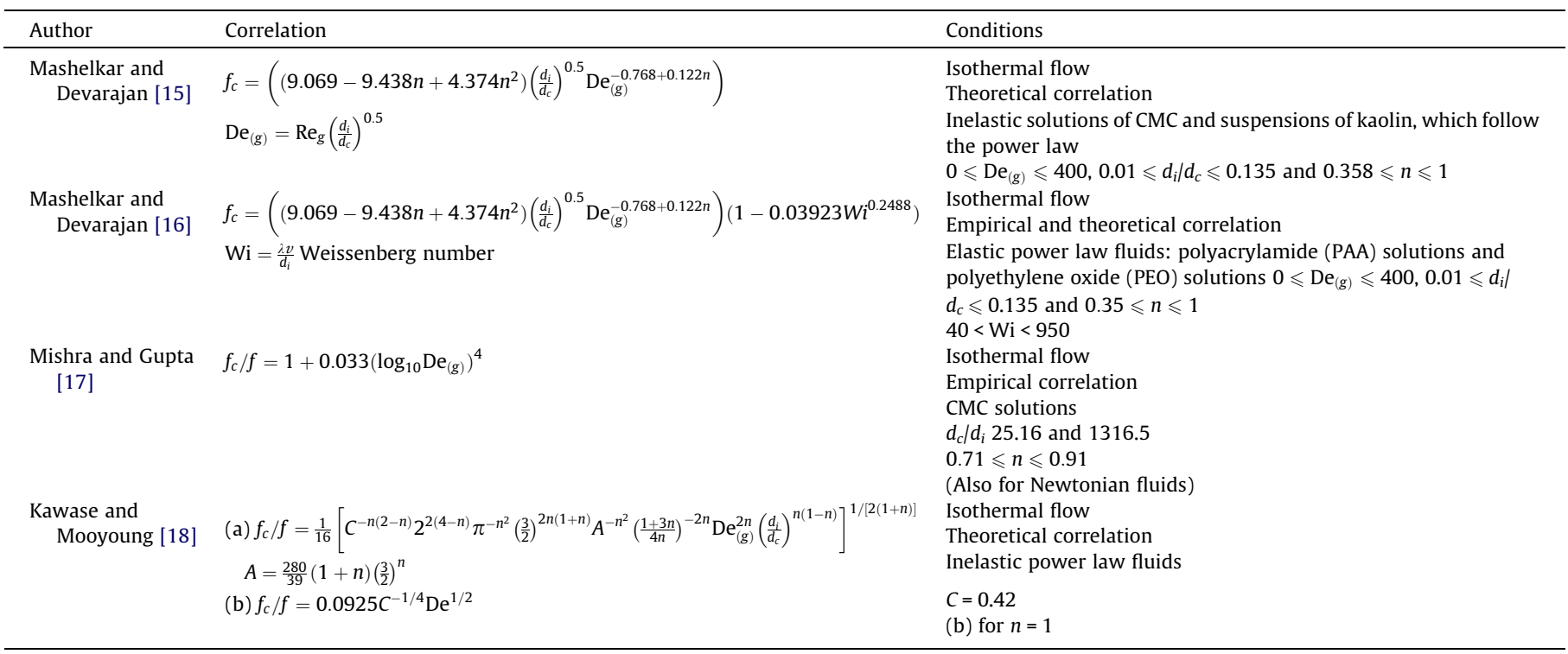

Table 3

Dimensions of the helical coil of copper.

\begin{tabular}{ll}
\hline & Average of the measured values \\
\hline Internal coil diameter $(\mathrm{mm})$ & $167.79 \pm 1.21$ \\
External coil diameter $(\mathrm{mm})$ & $179.47 \pm 1.27$ \\
Coil height $(\mathrm{mm})$ & $111.11 \pm 0.85$ \\
Distance between turns $(\mathrm{mm})$ & $4.99 \pm 0.09$ \\
Angle of the turns $\left(^{\circ}\right)$ & $3.17 \pm 0.19$ \\
Inside diameter of the tube $(\mathrm{mm})$ & $4.32 \pm 0.05$ \\
External diameter of the tube $(\mathrm{mm})$ & $6.35 \pm 0.05$ \\
Internal diameter of the fittings $(\mathrm{mm})$ & $4.01 \pm 0.03$ \\
Length of the coil tube $(\mathrm{m})$ & 5.5 \\
Coil pitch $(\mathrm{mm})$ & 11.34 \\
Number of turns & 9.4 \\
\hline
\end{tabular}

the cooling of the bath, by water flowing in the coil, in unsteady conditions:

$-\left(m_{\text {syst }} C p_{\text {syst }}\right) \frac{d T_{\text {oil }}}{d t}-(U A)_{\text {losses }}\left(T_{\text {oil }}-T_{\text {amb }}\right)=\dot{m}_{\text {water }} C p_{\text {water }}\left(T_{s}-T_{e}\right)$
The Fig. 2 shows the results of the experimental rig validation.

\subsection{Characterization of the fluids}

The Newtonians fluids were aqueous solutions of glycerol ( $w /$ w) of $25 \%, 36 \%, 43 \%, 59 \%$ and $78 \%$ and the non-Newtonians fluids were aqueous solutions of carboxymethylcellulose (CMC), $0.1 \%$, $0.2 \%, 0.3 \%, 0.4 \%$ and $0.6 \%(\mathrm{w} / \mathrm{w})$, with molar mass $3 \times 10^{5} \mathrm{~kg} \mathrm{kmol}^{-1}$ (Hercules 7H4C), and aqueous solutions of xanthan gum (XG), $0.1 \%$ and $0.2 \%(\mathrm{w} / \mathrm{w})$, with molar mass $2 \times 10^{6} \mathrm{~kg} \mathrm{kmol}^{-1}$ (Kelko keltrol TF). The values of the physical properties of the glycerol solutions were obtained through literature and the determination of the viscosity was done in a rotational viscometer. The physical properties of the non-Newtonian solutions, except the rheological properties, were taken as identical to those of pure water [20-22].

\subsubsection{Rheological characterization of the non-Newtonians fluids}

The viscous and elastic components of the non-Newtonian solutions were characterized in a cutting edge rheometer mark

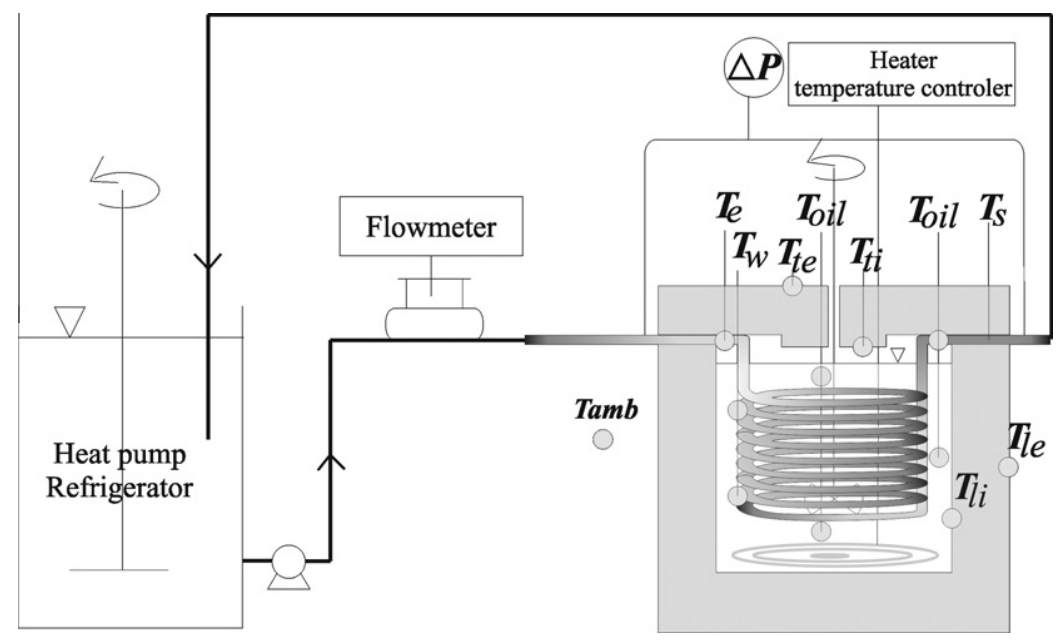

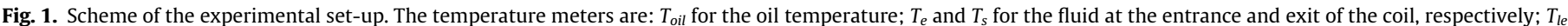

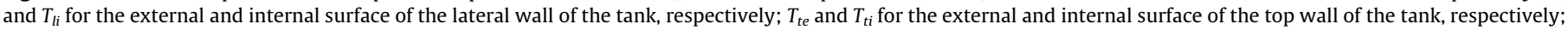
and $T_{a m b}$ for the ambient temperature; $\Delta P$ is the pressure drop of the fluid inside the helical coil. 


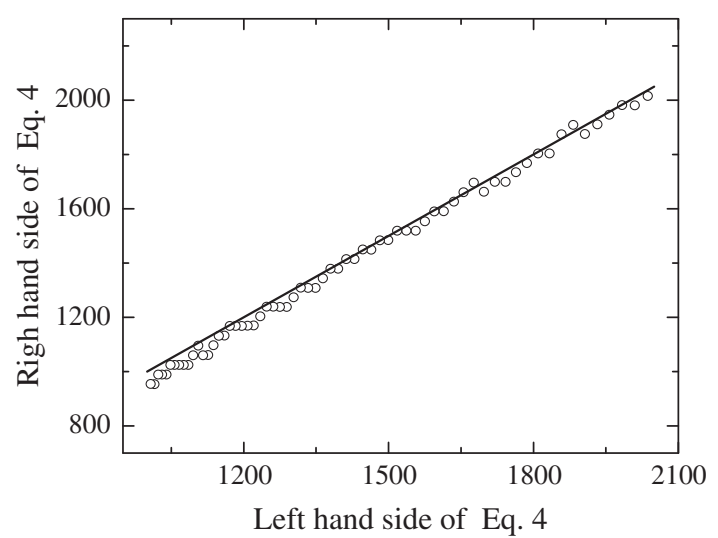

Fig. 2. Results of the experimental energy balance, Eq. (2), for rig validation; the line is at $45^{\circ}$.

PHYSICA model MCR30 and the geometry used was that of coneplate. The viscometric viscosity was determined as a function of the shear rate through shear tests at steady state and the elastic component through the dissipation module $\left(G^{\prime \prime}\right)$ and the storage module $\left(G^{\prime}\right)$ in oscillatory cutting tests.

The rheological tests were performed at temperatures between $20^{\circ} \mathrm{C}$ and $40^{\circ} \mathrm{C}$, according to the range of temperature of the fluids flowing inside the coil. It was used the method of reduced properties described by Bird et al. [23] to obtain the shift factor, $a_{T}$. This factor was calculated for each of the solutions and enables the knowledge of the rheological properties at a desired temperature knowing the properties at a reference temperature $\left(T_{0}\right)$.

The fluids studied exhibited shear-thinning behavior, well represented by the power law model in the range of shear rates used to calculate the friction factor, and behavior index $(n)$ independent of the temperature.

Figs. 3-6 present some of the results of the tests done to obtain the parameters of the power law (viscous component) and of the relaxation time (elastic component). Figs. 3 and 4 present, for $20^{\circ} \mathrm{C}$, the shear-thinning behavior of all solutions of CMC and XG studied, respectively. Figs. 5 and 6 show the variation of the storage and loss modules with the angular velocity for $0.3 \%(\mathrm{w} / \mathrm{w})$ CMC and $0.2 \%(\mathrm{w} / \mathrm{w})$ XG solutions. Furthermore, they illustrate the variation of these modules with the temperature. Table 4 shows the results of the power law parameters ( $n$ and $K$ ) for all solutions studied and the ranges where they are valid. Table 5 shows the relaxation times obtained from the loss $\left(G^{\prime}\right)$ and storage

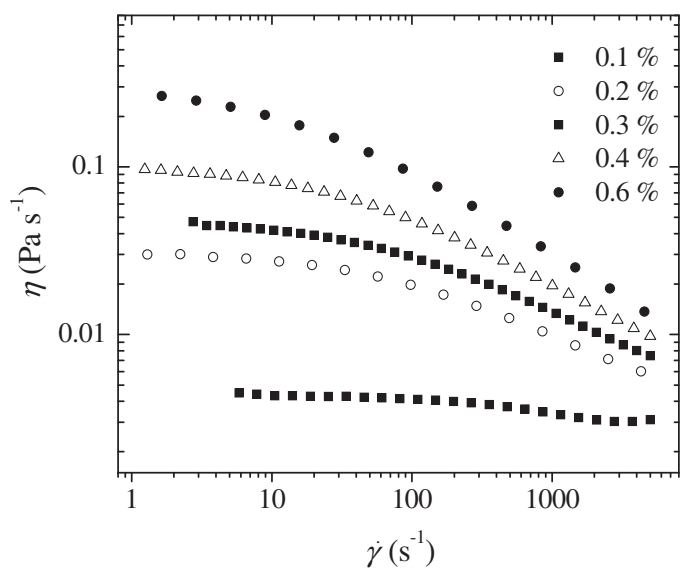

Fig. 3. Viscometricviscosity $(\eta) v s$. shear rate $(\dot{\gamma})$ for the solutions $0.1 \%, 0.2 \%, 0.3 \%$, $0.4 \%, 0.6 \% \mathrm{CMC}$ at $20^{\circ} \mathrm{C}$.

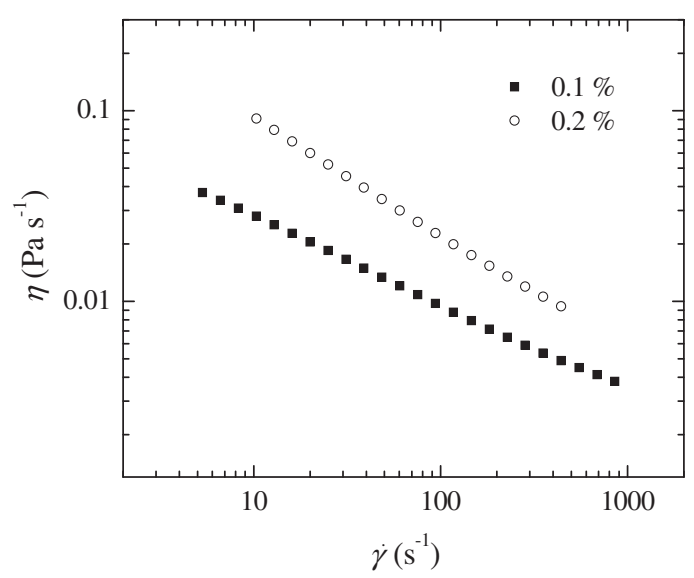

Fig. 4. Viscometric viscosity $(\eta)$ vs. shear rate $(\dot{\gamma})$ for the solutions $0.1 \%$ and $0.2 \% \mathrm{XC}$ at $20^{\circ} \mathrm{C}$.

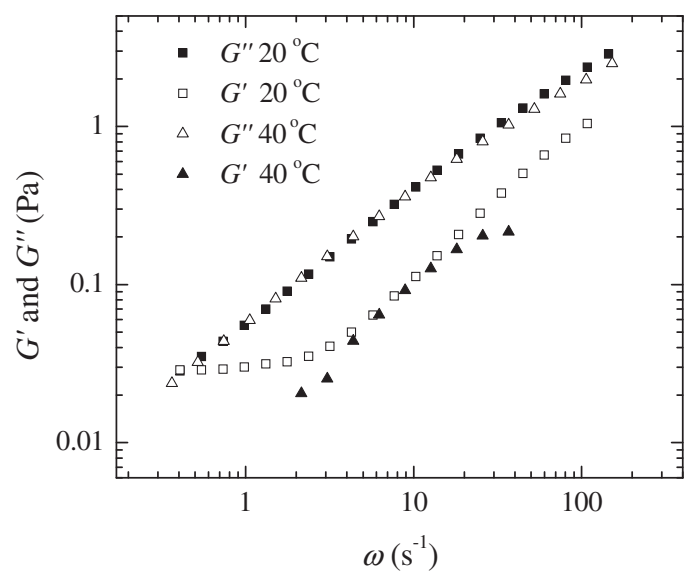

Fig. 5. Loss $\left(G^{\prime \prime}\right)$ and storage $\left(G^{\prime}\right)$ modules $v s$. angular frequency $(\omega)$ from oscillatory tests, for the solution $0.3 \%(\mathrm{w} / \mathrm{w}) \mathrm{CMC}, 20$ and $40{ }^{\circ} \mathrm{C}$.

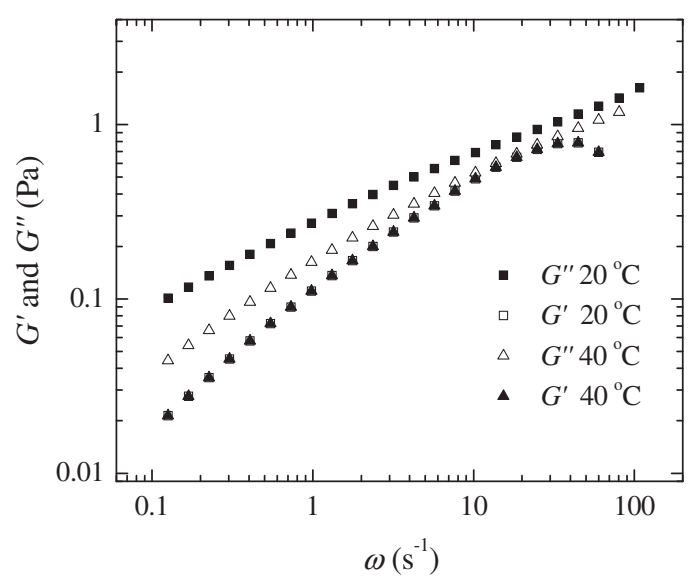

Fig. 6. Loss $\left(G^{\prime \prime}\right)$ and storage $\left(G^{\prime}\right)$ modules $v s$. angular frequency $(\omega)$ from oscillatory tests, for solution $0.2 \%(\mathrm{w} / \mathrm{w}) \mathrm{XG}, 20$ and $40^{\circ} \mathrm{C}$.

$\left(G^{\prime \prime}\right)$ modules at the reference temperature $\left(T_{0}\right)$ and also data from creep tests, these, obtained by Coelho and Pinho [24]. As shown in Table 5, the elasticity increases with the concentration of the solutions and it is greater for the solutions of XG. This increase can be verified in Figs. 5 and 6 where it is observed an approach of the values of the modules $G^{\prime}$ and $G^{\prime \prime}$ for the solution $0.2 \%$ of XG. 
Table 4

Consistency and behavior indexes for the solutions of CMC and dXG.

\begin{tabular}{|c|c|c|c|c|c|}
\hline & Solutions (\%) (w/w) & $K$ a $20^{\circ} \mathrm{C}\left(\mathrm{Pa} \mathrm{s}^{n}\right)$ & $K$ a $40{ }^{\circ} \mathrm{C}\left(\mathrm{Pa} \mathrm{s}^{n}\right)$ & $n$ & Range of $\dot{\gamma}\left(\mathrm{s}^{-1}\right)$ \\
\hline \multirow[t]{5}{*}{ CMC } & 0.1 & 0.008 & 0.004 & 0.90 & $100-4000$ \\
\hline & 0.2 & 0.082 & 0.045 & 0.70 & \\
\hline & 0.3 & 0.189 & 0.109 & 0.63 & \\
\hline & 0.4 & 0.376 & 0.227 & 0.58 & \\
\hline & 0.6 & 1.005 & 0.615 & 0.52 & \\
\hline \multirow[t]{2}{*}{$X G$} & 0.1 & 0.079 & 0.047 & 0.55 & $10-1000$ \\
\hline & 0.2 & 0.370 & 0.255 & 0.39 & \\
\hline
\end{tabular}

Table 5

Relaxation time for CMC and XG solutions at the reference temperature $T_{0}=30^{\circ} \mathrm{C}$.

\begin{tabular}{llllllll}
\hline & CMC & & & \multicolumn{5}{c}{ XG } \\
\hline $\begin{array}{c}\text { Solutions (\%) } \\
(w / w) \\
\begin{array}{c}\text { Relaxation time } \lambda \\
(\mathrm{s})\end{array}\end{array}$ & 0.1 & 0.2 & 0.3 & 0.4 & 0.6 & 0.1 & 0.2 \\
$\quad$ & & 0.162 & 0.346 & 0.488 & 1.086 & 2.933 & 3.419 \\
\hline
\end{tabular}

\subsection{Experimental conditions}

Table 6 shows the ranges of Reynolds, Dean, Prandtl and Helical numbers for the different glycerol solutions. For all the studied conditions, the minimum ( $\mathrm{m}$ ) and maximum (M) heat fluxes between the oil and the flowing glycerol solutions were, respectively, 67 and $131 \mathrm{~kW} \mathrm{~m}^{-2}$.

For the CMC and XG solutions, the range of the generalized Reynolds, $\mathrm{Re}_{\mathrm{g}}$, modified Dean, $\mathrm{De}_{(\mathrm{g})}$, modified Prandtl, $\operatorname{Pr}_{(g)}$, and Weissenberg, Wi, numbers are presented in Tables 7 and 8. For all the CMC solutions the minimum ( $\mathrm{m}$ ) and maximum (M) heat fluxes between the oil and the solution were, respectively, 2.5 and $11 \mathrm{~kW} \mathrm{~m}^{-2}$ and for the XG solutions, 4 and $8 \mathrm{~kW} \mathrm{~m}^{-2}$.

The generalized Reynolds number and modified Prandtl, $\operatorname{Pr}_{(g)}$ which are going to be referred along this work are defined as follows:

$\operatorname{Re}_{g}=\frac{d_{i}^{n} v^{2-n} \rho T_{0}}{a_{T}^{n} K\left(\frac{1+3 n}{4 n}\right)^{n} 8^{n-1} T}$

$\operatorname{Pr}_{(g)}=\frac{C_{p}}{k_{f}} K\left(\frac{v}{d_{i}}\right)^{n-1}\left(\frac{3 n+1}{4 n}\right)^{n} 8^{n-1} a_{T}^{n} \frac{T}{T_{0}}$

where $C_{p}$ and $k_{f}$, are, respectively, the heat capacity and the thermal conductivity of the fluid.

The dimensionless numbers presented in Tables 6-8 were obtained from 350 different experimental conditions.

\subsection{Friction factor, $f_{c}$, calculation method}

The friction factor for the fluid in circulation inside the coil, $f_{c}$, was calculated with the following equation:
$f_{c}=\frac{\Delta P d_{i}^{5} \rho \pi^{2}}{32 L_{\text {coil }} \dot{m}^{2}}$

where $\dot{m}$ is the fluid mass flow and $L_{\text {coil }}$ the length of the coil.

The fluid properties were determined at the mean temperature, $T_{m}$, between the temperature of the fluid at the entrance, $T_{e}$, and exit, $T_{s}$, of the coil, Eq. (8), and at the mean film temperature, $T_{f}$, between the internal temperature of the coil and the mean temperature $T_{m}$, Eq. (9):

$T_{m}=\frac{T_{e}+T_{s}}{2}$

$T_{f}=\frac{T_{w}+T_{m}}{2}$

The internal temperature of the coil was taken equal to the temperature of the oil of the bath; very low resistance to the heat flux in the oil and a very conductive material (coil of cooper). The value of the critical Reynolds number, $\operatorname{Re}_{c}$, used to define the transition from laminar to turbulent flow inside the coil was 6240 according to Ito [10].

The velocity and temperature profiles at the entrance of the coil are in development, according to Nigam et al. [25], along a distance equal to 30 diameters of the tube, i.e., $18 \%$ of the total length of the coil. However, the development of these profiles is not as in straight tubes, they suffer cyclic oscillations around the final velocity and temperature profiles before stabilization [25]. These inlet effects on the friction lost coefficient were neglected in the present study.

\section{Results}

\subsection{Newtonian fluids}

The friction factors were obtained experimentally, Eq. (7), using the mean temperature $\left(T_{m}\right)$, Eq. (8), and the mean film temperature $\left(T_{f}\right)$, Eq. (9), to determine the physical properties of the fluids. The results obtained for all the glycerol solutions and the friction factor for a straight tube are presented in Fig. 7.

In Figs. 8 and 9, the experimental data obtained for the friction factor of the glycerol solutions are compared, respectively, with the

Table 6

Reynolds, Dean, Prandtl and Helical numbers for the glycerol solutions.

\begin{tabular}{|c|c|c|c|c|c|c|}
\hline \multicolumn{2}{|c|}{ Glycerol solutions \% (w/w) } & \multirow{2}{*}{$\frac{78}{45}$} & \multirow{2}{*}{$\frac{59}{195}$} & \multirow{2}{*}{$\frac{43}{423}$} & \multirow{2}{*}{$\frac{36}{547}$} & \multirow{2}{*}{$\frac{25}{765}$} \\
\hline $\operatorname{Re}$ & $\mathrm{m}$ & & & & & \\
\hline & M & 357 & 1588 & 4159 & 6022 & 6293 \\
\hline \multirow[t]{2}{*}{ De } & $\mathrm{m}$ & 7 & 32 & 69 & 89 & 124 \\
\hline & M & 58 & 257 & 674 & 976 & 1020 \\
\hline \multirow[t]{2}{*}{$\operatorname{Pr}$} & $\mathrm{m}$ & 160 & 39 & 20 & 14 & 10 \\
\hline & M & 353 & 75 & 32 & 22 & 16 \\
\hline \multirow[t]{2}{*}{$\mathrm{He}$} & $\mathrm{m}$ & 7 & 32 & 69 & 89 & 124 \\
\hline & M & 58 & 257 & 674 & 976 & 1020 \\
\hline
\end{tabular}


Table 7

Generalized Reynolds, modified Dean, modified Prandtl and Weissenberg numbers for the CMC solutions.

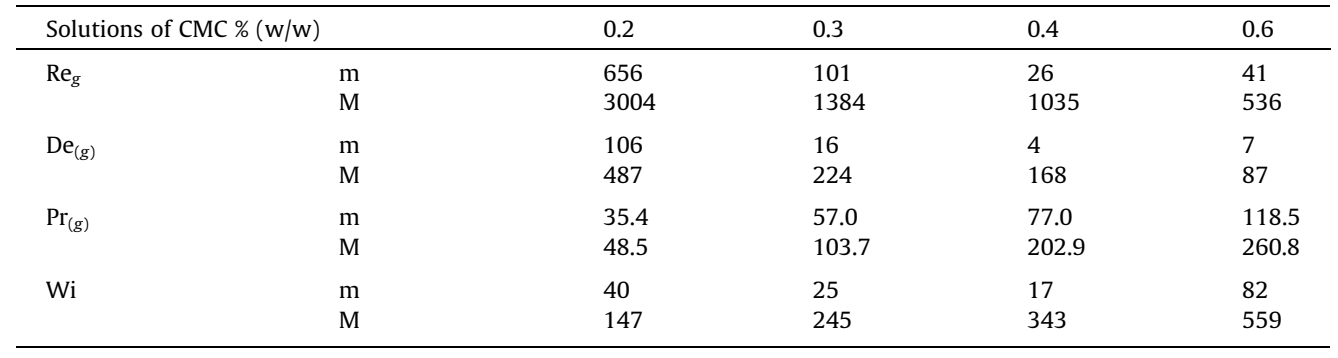

Table 8

Generalized Reynolds, modified Dean, modified Prandtl and Weissenberg numbers for XG solutions.

\begin{tabular}{llll}
\hline Solutions of XG \% (w/w) & & 0.1 & 0.2 \\
\hline $\operatorname{Re}_{g}$ & $\mathrm{~m}$ & 412 & 207 \\
& $\mathrm{M}$ & 6759 & 4873 \\
$\operatorname{De}_{(g)}$ & $\mathrm{m}$ & 67 & 34 \\
& $\mathrm{M}$ & 1096 & 790 \\
$\operatorname{Pr}_{(g)}$ & $\mathrm{m}$ & 11.0 & 17.0 \\
& $\mathrm{M}$ & 25.0 & 50.0 \\
$\mathrm{Wi}$ & $\mathrm{m}$ & 90 & 100 \\
& $\mathrm{M}$ & 865 & 988 \\
\hline
\end{tabular}

data of Hart et al. [14], physical properties determined at $T_{m}$ and of Schmidt [8], physical properties determined at $T_{f}$.

Table 9 shows the mean deviation, and standard deviation, between the experimental values of this work and the values obtained with the selected literature correlations.

Among the correlations in Table 9, it was chosen that of Hart et al. [14] to fit the experimental data of this work. The equation obtained, using the tool Solver/Excel, is:

$f_{c}=f\left[1+\frac{a \mathrm{De}^{b}}{70+\mathrm{De}}\right]$

with $a$ and $b$, respectively, 0.028 and 1.68 .

Hart [14] correlation was selected because: it covers the entire laminar flow range; when the Dean number tends to zero, the friction factor tends to the observed value in a straight tube (f); and the mean error in Table 9 is low. The fitting equation is important to compare the results of Newtonians fluids with those with CMC and XG solutions obtained in identical experimental conditions.

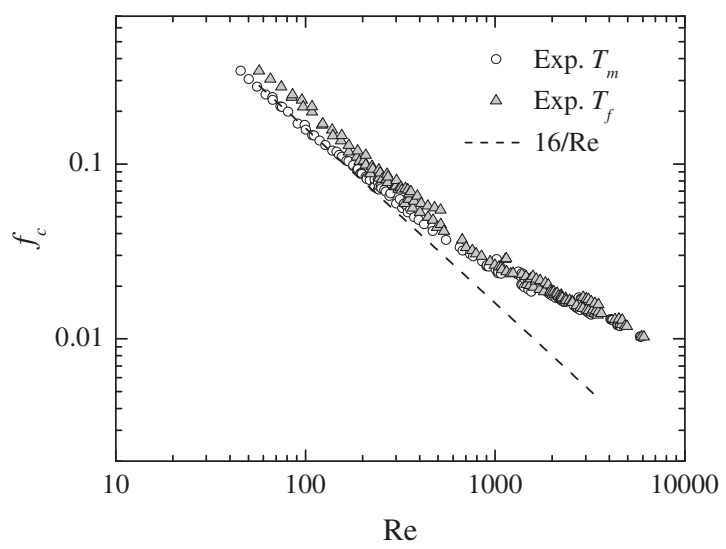

Fig. 7. Experimental friction factor $\left(f_{c}\right) v s$. Reynolds number (Re) for all the glycerol solutions determined at $T_{m}$ and $T_{f}$ for the helical coil and for a straight tube $(f=16)$ Re).

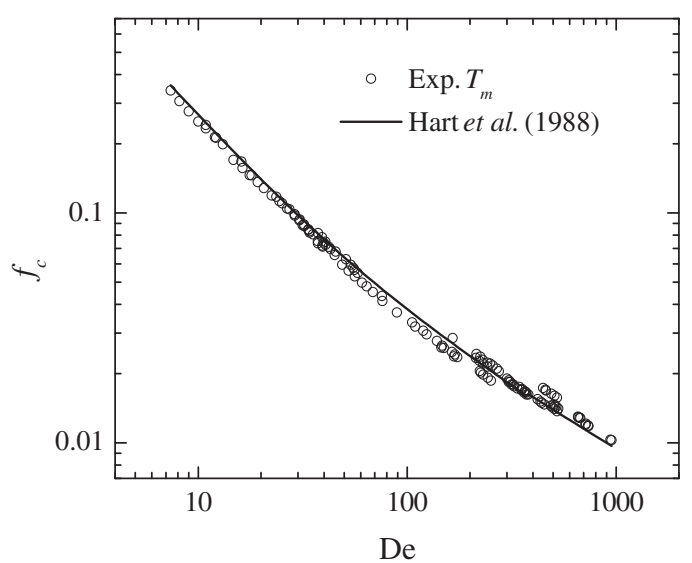

Fig. 8. Friction factor $\left(f_{c}\right) v s$. Dean number (De). Comparison between data from glycerol solution experiments $\left(T_{m}\right)$ with data given by Hart et al. [14].

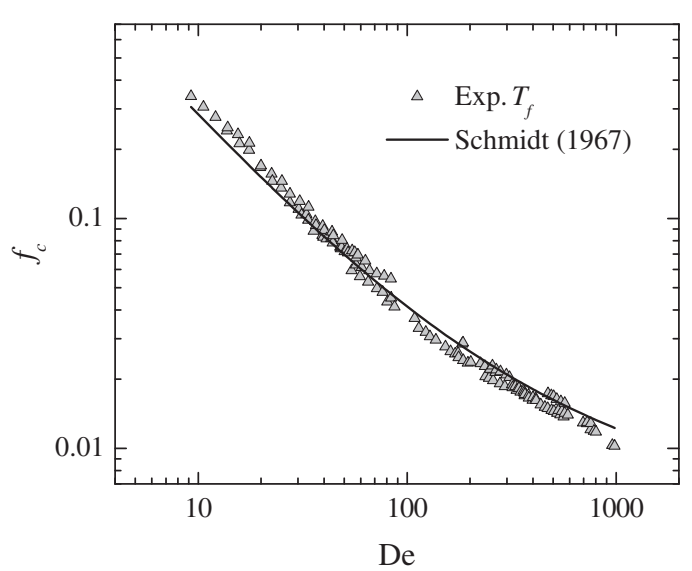

Fig. 9. Friction factor $\left(f_{c}\right) v s$. Dean number (De). Comparison between data from glycerol solution experiments $\left(T_{f}\right)$ with data given by Schmidt [8].

Table 9

Mean and standard deviation of the difference between experimental friction factor data and data obtained from the correlations in literature for glycerol solutions.

\begin{tabular}{lc}
\hline & Mean \pm standard deviation (\%) \\
\hline White [9] & $4.7 \pm 3.8$ \\
Ito [10] & $6.0 \pm 4.1$ \\
Mori and Nakayama [11] & $11.0 \pm 9.0$ \\
Schmidt [8] & $8.6 \pm 4.6$ \\
Tarbell and Samuels [12] & $8.0 \pm 3.7$ \\
Manlapaz and Churchill [13] & $6.0 \pm 4.9$ \\
Hart et al. [14] & $5.0 \pm 3.7$ \\
\hline
\end{tabular}


It will be designated from now on by Modified Hart Correlation (MHC).

Fig. 10 represents the fit of the experimental values, taking into account the study of Ali [4].

\subsection{Non-Newtonian fluids}

Fig. 11 compares data from the correlations selected in the literature for non-Newtonian fluids $[17,15,18]$ with predictions of MHC, Eq. (10). Figs. 12-17 present the experimental results and their comparison with data from the correlations selected in the literature for non-Newtonian fluids. Fig. 18 compares the friction factors, for all CMC and XG solutions, calculated with physical properties at the mean temperature and at the mean film temperature. Fig. 19 shows the study of Ali (2001) [4] applied to CMC, XG solutions and also MHC data.

\subsection{Uncertainty analysis of the results}

The mean and standard deviations of the relative uncertainties of all the experimental friction factors, Newtonian and non-Newtonian fluids, are $5.60 \pm 0.20 \%$. This uncertainty includes the variable physical properties corrections due to heating effects.

\section{Discussion of the results}

\subsection{Newtonians fluids}

Fig. 7 shows the experimental data of the friction factor $v s$. Reynolds number for the solutions of glycerol; physical properties calculated at the mean temperature of the fluid, $T_{m}$, and also at the mean temperature of the film, $T_{f}$. It can be observed that for $T_{m}$ and values of the Reynolds number lower than 120 ( $\mathrm{De}=20)$, the friction factors, for the helical coil, are very similar to those in a straight tube, i.e., the viscous forces determined the flow pattern and the effect of the centrifugal force is not noticeable. For Reynolds number greater than 120 , the friction factor values, for the helical coil, start to deviate to higher values. The deviation rate increases for Reynolds numbers higher than, approximately, 500 . This higher deviation rate is justified by the increasing effect of the centrifugal force in the flow pattern. Still in Fig. 7, it can be observed that for Reynolds number less than 190 ( $\mathrm{De}=30$ ), the friction factor values calculated at $T_{f}$ are higher than those at $T_{m}$. Furthermore, for Dean number greater than 30, the values are practically independent of the temperature chosen to calculate the

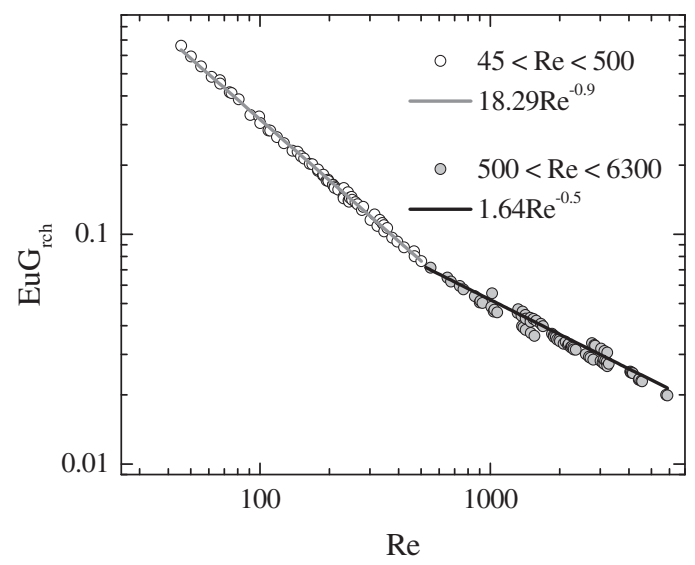

Fig. 10. Product of the Euler number (Eu) by the geometric number $\left(\mathrm{G}_{\mathrm{rhc}}\right) v s$. Reynolds number (Re) for the experimental results of the glycerol solutions at $T_{m}$. The fit equations are in accordance to Ali [4]. physical properties. Once more, this behavior stresses the importance of the viscous forces in the flow for low Reynolds number.

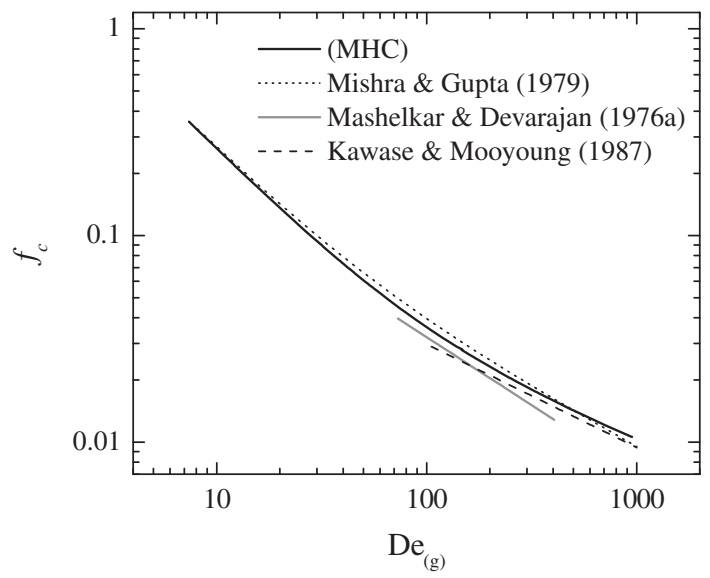

Fig. 11. Friction factors $\left(f_{c}\right)$ vs. generalized Dean number $\left(D e_{g}\right)$ for non-Newtonians fluids based on the works of Mishra and Gupta [17], Mashelkar and Devarajan [15] and Kawase and Mooyoung [18]. Comparison with MHC predictions.

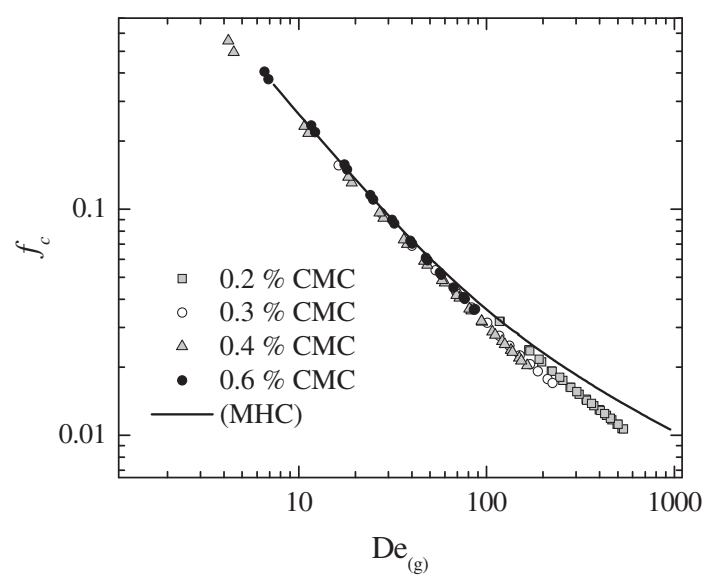

Fig. 12. Experimental friction values $\left(f_{c}\right) v s$. generalized Dean number $\left(D e_{g}\right)$, with physical properties at $T_{m}$, for $0.2 \%, 0.3 \%, 0.4 \%$ and $0.6 \%(\mathrm{w} / \mathrm{w}) \mathrm{CMC}$ solutions and comparison with MHC predictions.

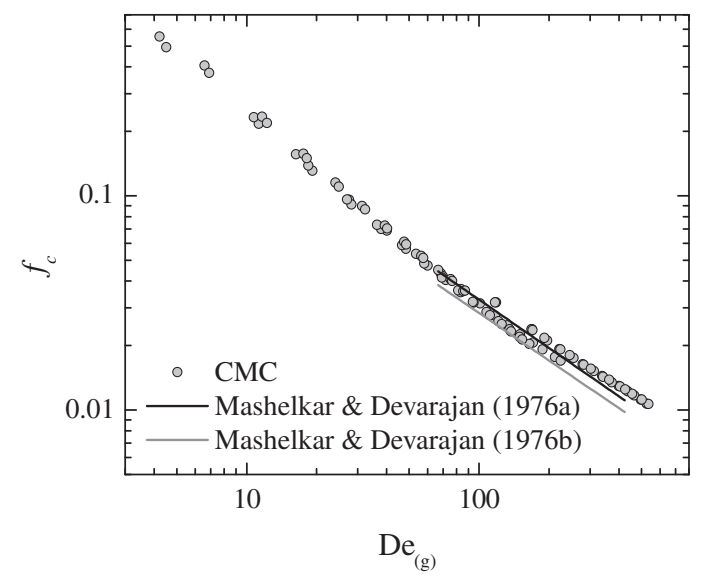

Fig. 13. Experimental friction values $\left(f_{c}\right) v s$. generalized Dean $\left(\mathrm{De}_{g}\right)$ number, with physical properties at $T_{m}$, for all CMC solutions and comparison with predictions from Mashelkar and Devarajan [15,16]. 


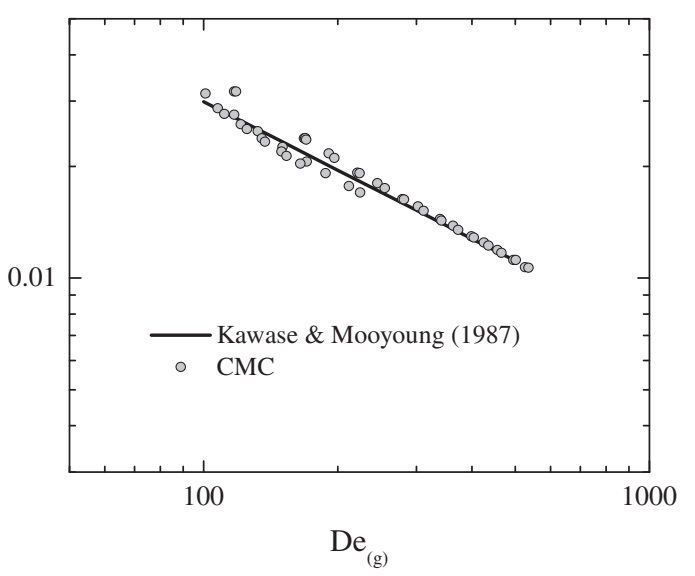

Fig. 14. Experimental friction values $\left(f_{c}\right)$ vs. generalized Dean number $\left(\mathrm{De}_{g}\right)$, with physical properties at $T_{m}$, for $0.2 \%, 0.3 \%$ and $0.4 \%(\mathrm{w} / \mathrm{w})$ CMC solutions and comparison with Kawase and Mooyoung [18] predictions.

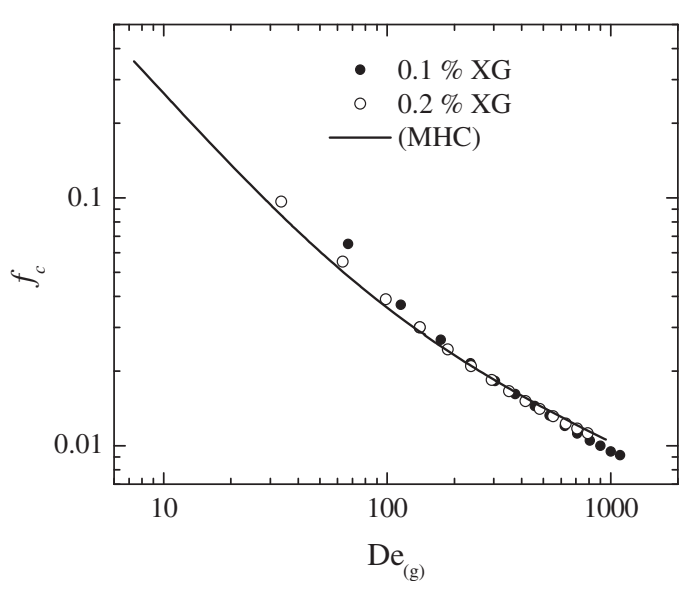

Fig. 15. Experimental friction values $\left(f_{c}\right)$ vs. generalized Dean number $\left(\mathrm{De}_{g}\right)$, with physical properties at $T_{m}$, for $0.1 \%$ and $0.2 \%(\mathrm{w} / \mathrm{w})$ solutions of XG and comparison with MHC predictions.

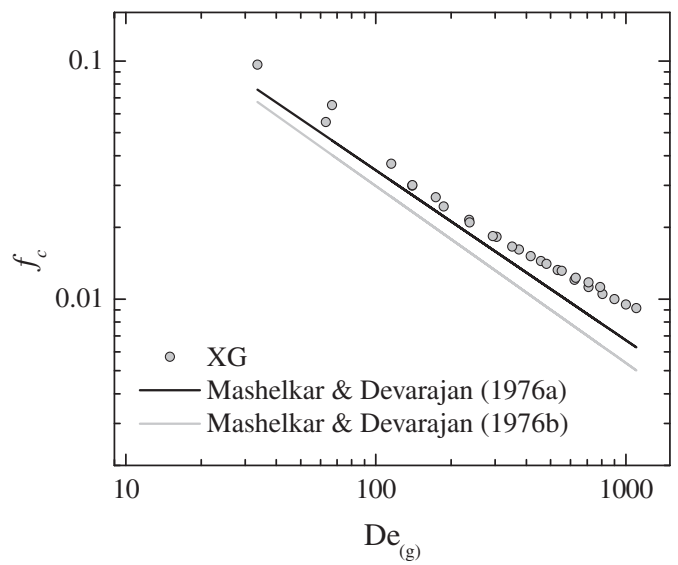

Fig. 16. Experimental friction values $\left(f_{c}\right)$ vs. generalized Dean number $\left(\mathrm{De}_{(g)}\right)$, with physical properties at $T_{m}$, for solutions of XG and comparison with Mashelkar and Devarajan $[15,16]$ predictions.

As shown in Table 9, the friction factor values obtained with glycerol solutions flowing with simultaneous heat transfer, constant wall temperature as boundary condition, are similar to those

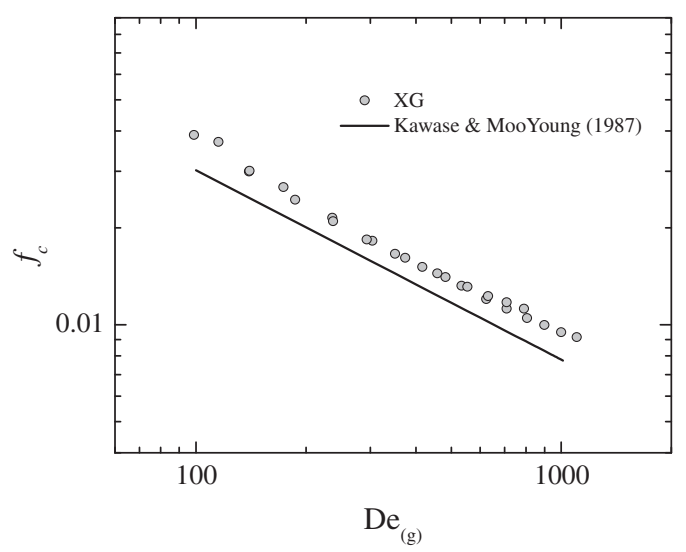

Fig. 17. Experimental friction values $\left(f_{c}\right) v s$. generalized Dean number $\left(\operatorname{De}_{(g)}\right)$, with physical properties at $T_{m}$, for $0.1 \%$ and $0.2 \%(\mathrm{w} / \mathrm{w})$ solutions of XG and comparison with Kawase and Mooyoung [18] predictions.

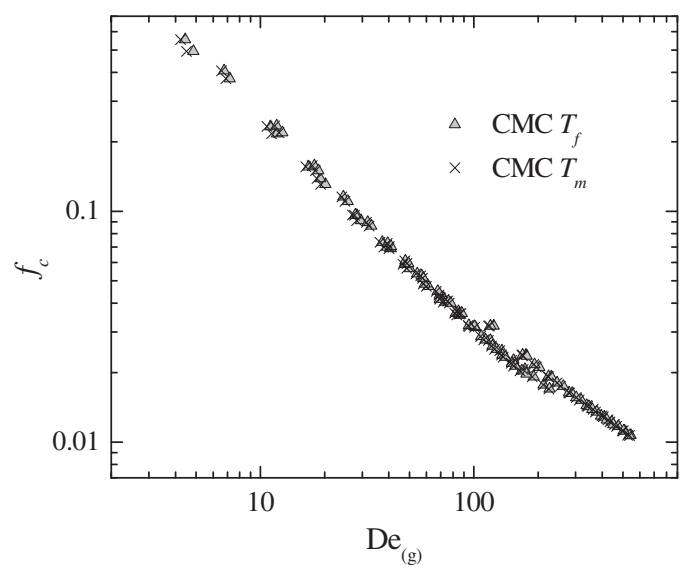

(a)

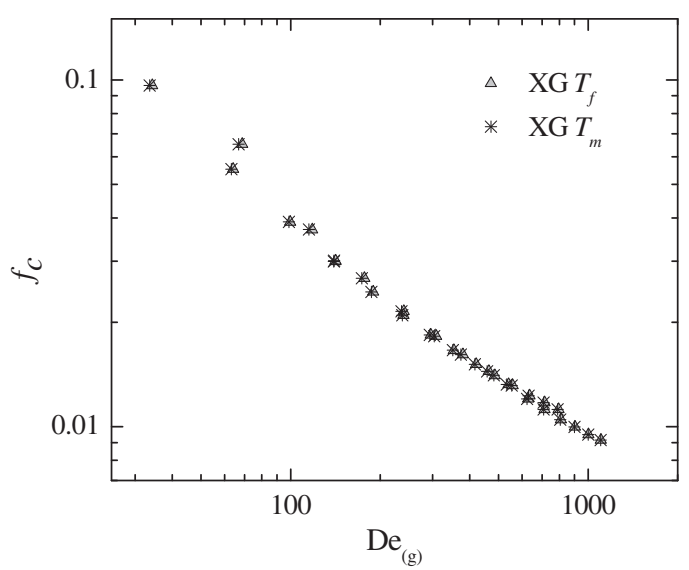

(b)

Fig. 18. Experimental friction factors $\left(f_{c}\right) v s$. generalized Dean number $\left(\operatorname{De}_{(g)}\right)$, with physical properties at $T_{m}$ and at $T_{f}$ for the solutions: (a) CMC and (b) XG.

obtained with the theoretical correlations of Mori Nakayama [11], Tarbell and Samuels [12] and Manlapaz and Churchill [13] and to those obtained with the experimental correlations of White [9], Ito [10] and Hart et al. [14]; all of them valid for isothermal flow and physical properties calculated at the mean temperature of the fluid. 


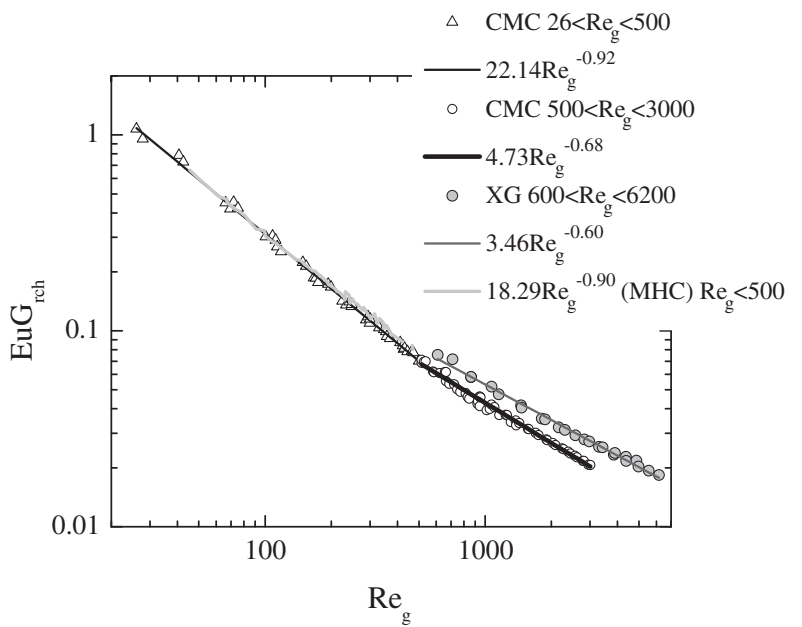

Fig. 19. Product of Euler number (Eu) by geometric number $\left(\mathrm{G}_{\mathrm{rhc}}\right)$ vs. generalized Reynolds number $\left(\operatorname{Re}_{g}\right)$ for all solutions of CMC and XG at $T_{m}$. Solid lines represent fit equations according to Ali [4].

Fig. 8 shows the comparison between the results obtained with the glycerol solutions and those from Hart et al. [14]. Although the correlation of Hart was obtained in isothermal conditions, the results are very similar. The reduced temperature increase of the fluid between the inlet and outlet of the coil justify this similarity. Therefore, the correlation of Hart was selected to fit the data of this work; the reasons were already explained above and the fitting equation, Eq. (10), has been designated by Modified Hart Correlation (MHC). The empirical correlation of Schmidt [8], whose results are shown in Fig. 9, is valid for simultaneous heat transfer at constant wall temperature (physical properties calculated at the mean temperature of the film). Although the boundary condition is the same, the mean deviation between the results of Schmidt and those of the present work is higher $(8.6 \pm 4.6 \%)$ than the mean deviation with Hart data $(5.0 \pm 3.7 \%)$.

Ali [4] refers that, in low laminar regime, the effect of the main axial flow on the pressure drop overlaps the effect of the secondary flow. When Reynolds number is higher than 500, once more following Ali, there is a continuous increase of the intensity of the secondary flow with Reynolds number and its effect in the pressure drop becomes appreciable. The increasing ratio, observed in Fig. 7, between the pressure drop in the coil and that in a straight tube, confirms these findings of Ali. In Fig. 10, the experimental data of this work are fitted with an equation of the type $\mathrm{EuG}_{\mathrm{rhc}}=a \mathrm{Re}^{-b}$ and the following conclusions can be taken:

- Ali' correlation for $\operatorname{Re}<500$ is $E u G_{r h c}=21.88 \operatorname{Re}^{-0.9}$ while the fitting equation has very similar coefficients, $\mathrm{EuG}_{\mathrm{rhc}}=18.29 \mathrm{Re}^{-0.9}$;

- Ali' correlation for $500<\operatorname{Re}<6300$ is $\mathrm{EuG}_{\mathrm{rhc}}=5.25 \mathrm{Re}^{-2 / 3}$ while the fitting equation has slightly different coefficients, $\mathrm{EuG}_{\mathrm{rhc}}=1.64 \mathrm{Re}^{-0.5}$.

In resume, the experimental data of the present work for Newtonians fluids are well represented by the Modified Hart Correlation (MHC) but they are also well represented by the equations presented by Ali [4] considering two regimes in the laminar range.

This behavior will be taken on account in the analysis of the non-Newtonian fluids data

\subsection{Non-Newtonians fluids}

The friction factors $\left(f_{c}\right)$ obtained for the solutions of glycerol are similar, Fig. 11, to those obtained with the experimental correlation of Mishra and Gupta [17], which is valid for Newtonian and also non-Newtonian shear-thinning fluids, with flow behavior index greater than 0.71 . The results of Rohsenow et al. [21] also confirm this agreement is. In Fig. 11, it can also be observed that the values of the friction factors obtained with the correlation of Mashelkar and Devarajan [15], valid for shear-thinning fluids, are lower than those from Mishra and Gupta, a deviation about 20\%. Still in Fig. 11, it is represented data from the work of Kawase and Mooyoung [18], a theoretical correlation valid for non-Newtonian fluids that follow the power law. These data are similar to those of Mashelkar and Devarajan [15], i.e., both lower than the results obtained with Mishra and Gupta correlation.

The experimental friction factors obtained with $\mathrm{CMC}$ solutions are of the order of magnitude of those obtained with the glycerol solutions until Dean numbers, based on a generalized Reynolds number, around 80 (Fig. 12). For values of $\mathrm{De}_{(g)}$ above 80 , the CMC values are progressively lower. The mean deviation is $10 \%$, with negligible deviations for $\mathrm{De}_{(g)}$ less than 80 and increasing deviations, to a maximum of $20 \%$, for values of $\mathrm{De}_{(\mathrm{g})}$ higher than 80 . According to these results, the shear thinning behavior seems to have increasing effect in the range of influence of the secondary flow.

The theoretical equation of Mashelkar and Devarajan [15] is valid for isothermal flow of inelastic non-Newtonian fluids that follow the power law and for $70<\mathrm{De}_{(\mathrm{g})}<400$. This equation depends on the flow behavior index and fits well the results of the solutions of CMC studied in this work (Fig. 13). The mean and standard deviations are $6.9 \pm 3.8 \%$.

The correlation of Mashelkar and Devarajan [16] accounts for the elasticity of the fluids, through the Weissenberg number, and provides a reduction of the friction factor values for increasing elasticity. As can be seen, also in Fig. 13, the results obtained with this correlation are lower than the experimental results of the CMC solutions, the mean and standard deviation of $11.6 \pm 4.9 \%$. The low elasticity behavior of the CMC solutions justifies this increase.

In Fig. 14 are compared the results obtained with the solutions of CMC with those obtained with the correlation of Kawase and Mooyoung [18] for non-Newtonian fluids. They are very similar, being the mean and standard deviations, for all concentrations, $3.2 \pm 1.4 \%$.

The experimental friction factors obtained with XG solutions are of the order of magnitude of those for the glycerol for all the range of the Dean number (Fig. 15).

Figs. 16 and 17 show the comparison between the experimental results obtained with XG solutions and those obtained with the correlations of Mashelkar and Devarajan [15], Mashelkar and Devarajan [16] and Kawase and Mooyoung [18]. The experimental data are higher, being the deviations respectively, $14.6 \pm 5.2 \%$, $31.5 \pm 5.2 \%$ and $19.2 \pm 2.1 \%$. It should be noted that the results of Mashelkar and Devarajan [16] were obtained in a range of Weissenberg numbers identical to those of the present work. These authors predicted a decrease of the friction factor with an increase of the viscoelasticity. For the XG solutions of this work, the opposite behavior was observed. The temperature effects on the flow pattern can be a plausible justification but a definitive answer can only be given after a detailed experimental analysis of the flow patterns of an elastic fluid inside the coil.

By comparing CMC and XG results, it is verified that they are similar until generalized Dean number around 80 and lower for values greater than 80 . The differences between the rheological properties of the solutions are the index of behavior, lower for the XG solutions as it is shown in Table 4, and the relaxation time, higher for the XG solutions as it is shown in Table 5 . The Weissenberg numbers, for these solutions, are in Tables 7 and 8 and the highest values are for $0.6 \% \mathrm{CMC}$ and XG solutions. Once more, the difference in the friction factors values appears when the centrifugal force becomes important in the development of the flow. 
Fig. 18 shows that the results are not sensitive to the temperature at which the physical properties of the solutions are determined $\left(T_{m}\right.$ and $\left.T_{f}\right)$. Again, it should be stressed the low difference between outlet and inlet temperatures in the coil.

Although the study of Ali [4], related to different regimes in laminar flow, was developed for Newtonian fluids, it was felt important to verify its application to non-Newtonian fluids. For generalized Reynolds numbers until $500\left(\mathrm{De}_{(\mathrm{g})}=80\right)$, the fit to the CMC experimental data is $\mathrm{EuG}_{\mathrm{rhc}}=22.14 \mathrm{Re}_{g}^{-0.92}$ and for $500<\mathrm{Re}_{g}<3004$ is $4.73 \mathrm{Re}_{g}^{-0.68}$. Both fits are close to the expressions presented by Ali for Newtonian fluids. For solutions of XG it was not possible, not enough data, to apply the study of Ali in the range, $\operatorname{Re}_{g}<500$. For the range $500<\mathrm{Re}_{g}<3004$ the expression fitted is $\mathrm{EuG}_{\mathrm{rhc}}=3.46 \mathrm{Re}_{g}^{-0.60}$.

Fig. 19 shows the application of Ali study to CMC and XG solutions. From this figure, it can be taken important inferences:

- for $\mathrm{Re}_{g}$ until $500\left(\mathrm{De}_{(g)}=80\right)$ the results for Newtonian and nonNewtonian fluids overlap;

- there is a change of the flow regime at $\operatorname{Re}_{g}$ of $500\left(\mathrm{De}_{(g)}=80\right)$ for CMC solutions and also for glycerol solutions.

- For $\mathrm{Re}_{\mathrm{g}}$ higher than 500 there is a different behavior of the CMC and $X G$ solutions. The friction factors of the XG solutions are higher but the functionality between $\mathrm{EuG}_{\mathrm{rhc}}$ and $\mathrm{Re}_{\mathrm{g}}$, for both solutions, is that proposed by Ali [4].

- According to the data, the friction factors in non-Newtonian fluids decrease with an increase of the shear thinning behavior and seems to increase when the elastic behavior increases.

The last inference should be tested with other fluids with different rheological behavior.

\section{Conclusions}

To calculate the friction factors for Newtonian fluids flowing in a helical coil with simultaneous heat transfer at constant wall temperature, one can use, with accuracy, the correlations in the literature for isothermal flow. No significant difference between the values calculated with the physical properties at the mean film temperature of the fluid $\left(T_{f}\right)$ and at the mean bulk temperature of the fluid $\left(T_{m}\right)$ was found.

The correlation of Hart et al. [14], valid for isothermal flow, fits well the experimental data.

Ali [4] observed two flow patterns along the laminar regime, with a critical Reynolds number at $500(\mathrm{De}=80)$. The Newtonian data of the present study confirm Ali conclusions even with heat transfer.

For non-Newtonian fluids the most important conclusions are:

- for modified Dean numbers less than 80 , the CMC results are similar to those obtained with Newtonian fluids;

- for modified Dean numbers greater than 80 the CMC results are lower than those obtained with Newtonian fluids, a maximum deviation of $20 \%$

- for $\mathrm{De}_{(g)}>80$, the CMC results are well represented by the theoretical correlation of Mashelkar and Devarajan [15], valid for isothermal flow and for fluids that follow the power law;

- for $\operatorname{De}_{(g)}>80$, the CMC results are well represented by Mashelkar and Devarajan [16] correlation valid for the same conditions of Mashelkar and Devarajan [15], but also taking in account the elasticity;

- for $\mathrm{De}_{(g)}>80$ the CMC results are well represented by the theoretical correlation of Kawase and Mooyoung [18] valid for inelastic fluids following the power law;

- the XG data, $\mathrm{De}_{(g)}>80$, are higher than the CMC data for the same range, so an increase of the elasticity seems to induce high friction factors, at least when there is simultaneous heat transfer;
- CMC and XG data are not dependent on the temperature at which the physical properties are determined $\left(T_{m}\right.$ and $\left.T_{f}\right)$.

The application of Ali [4] study to non-Newtonian fluids shows that for laminar flow there are two regimes with a critical generalized Reynolds number of $500\left(\mathrm{De}_{(g)}>80\right)$, the same value observed by Ali and also that obtained for the Newtonian solutions of the present work with heat transfer.

\section{Acknowledgements}

The authors acknowledge the support in this work of Eng. Víctor Ferreira of Institute of Mechanical Engineering and Industrial Management of Oporto and of Dr. Adélio Cavadas and Dr. Paulo Coelho of Faculty of Engineering University of Oporto.

\section{References}

[1] W.R. Dean, The stream-line motion of fluid in a curved pipe, Philos. Mag. 5 (1928) 673-695 (Second paper).

[2] Y. Zhou, S.N. Shah, Fluid flow in coiled tubing: a literature review and experimental investigation, J. Can. Petrol Technol. 43 (2004) 52-61.

[3] P. Naphon, S. Wongwises, A review of flow and heat transfer characteristics in curved tubes, Renew. Sustain. Energy Rev. 10 (2006) 463-490.

[4] S. Ali, Pressure drop correlations for flow through regular helical coil tubes, Fluid Dyn. Res. 28 (2001) 295-310.

[5] A. Cioncolini, L. Santini, An experimental investigation regarding the laminar to turbulent flow transition in helically coiled pipes, Experim. Therm. Fluid Sci. 30 (2006) 367-380.

[6] R.A. Seban, E.F. McLaughlin, Heat transfer in tube coils with laminar and turbulent flow, Int. J. Heat Mass Trans. 6 (1963) 387-395.

[7] G.F.C. Rogers, Y.R. Mayhew, Heat transfer and pressure loss in helically coiled tubes with turbulent flow, Int. J. Heat Mass Trans. 7 (1964) 1207-1216.

[8] E.F. Schmidt, Heat transfer and pressure loss in spiral tubes, Chem-Ing-Tech. 39 (1967) 781.

[9] C.M. White, Streamline flow through curved pipes, Proc. R. Soc. Lond. Ser. AContain. Pap. Math. Phys. Character. 123 (1929) 645-663.

[10] H. Ito, Laminar flow in curved pipes, Z. Angew Math. Mech. 49 (1969) 653.

[11] Y. Mori, W. Nakayama, Study on forced convective heat transfer in curved pipes (1st report, laminar region), Int. J. Heat Mass Trans. 8 (1965) 67-82.

[12] J.M. Tarbell, M.R. Samuels, Momentum and heat transfer in helical coils, The Chem. Eng. J. 5 (1973) 117-127.

[13] R.L. Manlapaz, S.W. Churchill, Fully-developed laminar convection from a helical-coil, Chem. Eng. Commun. 9 (1981) 185-200.

[14] J. Hart, J. Ellenberger, P.J. Hamersma, Single- and two-phase flow through helically coiled tubes, Chem. Eng. Sci. 43 (1988) 775-783.

[15] R.A. Mashelkar, G.V. Devarajan, Secondary flows of non-Newtonian fluids. 1. Laminar boundary-layer flow of a generalized non-newtonian fluid in a coiled tube, T. I. Chem. Eng-Lond. 54 (1976) 100-107.

[16] R.A. Mashelkar, G.V. Devarajan, Secondary flow of non-Newtonian fluids. 2 Frictional losses in laminar-flow of purely viscous and viscoelastic fluids through coiled tubes, T. I. Chem. Eng-Lond. 54 (1976) 108-114.

[17] P. Mishra, S.N. Gupta, Momentum-transfer in curved pipes. 2. Non-Newtonian fluids, Ind. Eng. Chem. Process Des. Develop. 18 (1979) 137-142.

[18] Y. Kawase, M. Mooyoung, Momentum and heat-transfer in non-Newtonian fluids flowing through coiled tubes, Ind. Eng. Chem. Res. 26 (1987) 1248 1254.

[19] A.B. Metzner, J.C. Reed, Flow of non-Newtonian fluids - correlation of the laminar, transition and turbulent-flow regions, AIChe J. 1 (1955) 434-440.

[20] F.T. Pinho, P.M. Coelho, Non-Newtonian heat transfer in rheology, in: Crispulo Gallegos (Ed.), Encyclopedia of Life Support Systems (EOLSS), Developed Under the Auspices of the UNESCO, Eolss Publishers, Oxford, UK, 2009 (Chapter 17).

[21] W.M. Rohsenow, Y.I. Cho, J.P. Hartnett, Handbook of Heat Transfer, third ed., Mcgraw-Hill Education-Europe, 1998.

[22] N. Semmar, J.L. Tanguier, M.O. Rigo, Analytical expressions of specific heat capacities for aqueous solutions of CMC and CPE, Thermochimica Acta. 419 (2004) 51-58.

[23] R.B. Bird, R.C. Armstrong, O. Hassager, Dynamics of Polymeric Liquids, Fluid Mechanics, second ed., John Wiley and Sons, New York, 1987.

[24] P.M. Coelho, F.T. Pinho, Vortex shedding in cylinder flow of shear-thinning fluids I. Identification and demarcation of flow regimes, J. Non-Newton. Fluid Mech. 110 (2003) 143-176.

[25] K.D.P. Nigam, S. Agarwal, V.K. Srivastava, Laminar convection of nonNewtonian fluids in the thermal entrance region of coiled circular tubes, Chem. Eng. J. 84 (2001) 223-237. 